\title{
SPECTROSCOPIC OBSERVATIONS OF Z ANDROMEDAE AND CI CYGNI FROM 1968 TO 1973
}

\author{
A. MAMMANO, L. ROSINO, and S. YILDIZDOGDU \\ Asiago Astrophysical Observatory, University of Padova
}

\begin{abstract}
A description is given of the spectral changes observed in the symbiotic stars $Z$ And and CI Cyg during the nova-like outbursts of 1968 and 1971-73. The observed features can be interpreted by assuming that transient shells are formed around the hot component of a binary system, imbedded in a small nebula.
\end{abstract}

A systematic spectroscopic survey of symbiotic stars has been carried on at Asiago since 1960 with the $122 \mathrm{~cm}$ telescope. Several spectrographs and combinations were used in the survey, with dispersion ranging from 40 to $240 \AA \mathrm{mm}^{-1}$ at $\mathrm{H} \gamma$. The present communication gives a short description of the spectral variations observed in the symbiotic stars Z And and CI Cyg during a period of intense activity from 1968 to the present. A more detailed study is being prepared.

Most of the spectra described here have been obtained with the one-prism spectrograph at the Cassegrain focus of the $122 \mathrm{~cm}$ reflector, with the following combinations: Camera VI, with S20 Carnegie Image Tube $\left(60 \AA \mathrm{mm}^{-1}\right.$ at $\left.\mathrm{H} \gamma\right)$; Camera VII, with Sl Carnegie infrared Image Tube (dispersion at $\lambda 8000 \AA, 365 \AA \mathrm{mm}^{-1}$ ). Some spectra of CI Cyg were also taken with the grating spectrograph at the Newtonian focus, with S25 Westinghouse intensifier (dispersion: $120 \AA \mathrm{mm}^{-1}$ ).

\section{1. $Z$ Andromedae}

The star underwent a nova-like outburst in 1968. The observations here reported cover the post-maximum period from January 1969, when the star was declining to minimum, up to 1973. In 1971 the star, which was about $10^{m} 2$, slowly declined to $10^{m} 7$, rising again to $10^{m} 0$ at the end of the year and then to 10.4 in June 1972. Since that time the variable showed only slow fluctuations of small amplitude.

Five spectra, taken in 1969, January 6 and October 8; 1970, November 4;1972, October 30, and 1973, October 10 with the Camera VI have been examined, together with some infrared spectra obtained during the same period. From 1969 to 1972 the spectra look remarkably similar. They show (Figure 1) strong absorption bands of TiO over an intense continuum. The emission lines are sharp. In 1969 the strongest emission lines were: Balmer lines $\mathrm{H} \alpha$ through $\mathrm{H} \varepsilon$; He II $4686 \AA$ stronger than $\mathrm{H} \gamma$; He I lines at 4471, 4922, 5015, 5876 very strong, 6678, 7065 and $10830 \AA$. The [O III] nebular lines 4363, 4959, $5007 \AA$ were rather weak. Other He II lines together with lines of $\mathrm{C} \mathrm{IV},[\mathrm{Fe} \mathrm{VI}]$ and $[\mathrm{Fe} \mathrm{VII}]$ were indicative of a very high degree of ionization. At the same time, the presence of fairly strong permitted and forbidden lines of $\mathrm{Fe}$ II indicated to a contrasting solution: that of a relatively low excitation.

No conspicuous changes were seen during the following years up to 1972, although 


\section{$Z$ AND}

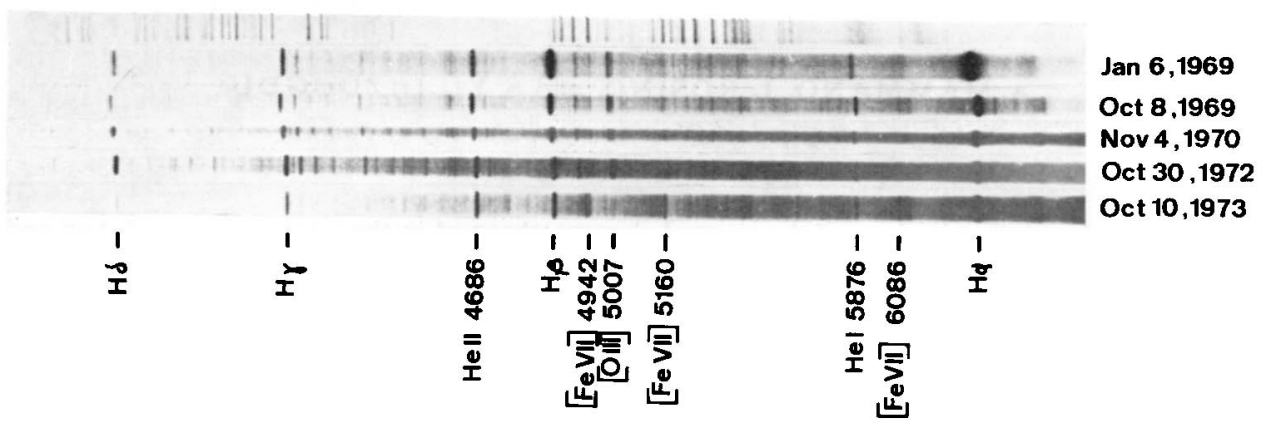

Fig. 1. Spectra of Z And from 1969 to 1973.

there were some indications of an increasing excitation, with moderate strengthening of [Fe VI] and [Fe VII]. The rising of excitation, on the whole, was quite apparent in 1973: while $\mathrm{Fe}$ II and [O III] lines weakened, [ $\mathrm{Fe} \mathrm{VI}] 5676$ and particularly [Fe VII] 4942, 5160, and 6086 clearly strengthened. At the same time there was an enhancement of the absorption band spectrum, which was well extended to all the visible region. The infrared was dominated by continuous emission and by absorption bands of TiO.

\section{CI Cygni}

Four maxima have been recorded in the light curve of CI Cyg: in 1911, 1937, 1971 and 1973 (Beljakina, 1974). The last two outbursts have been covered spectroscopically at Asiago, while no spectroscopic data are available for the oldest two.

In 1969 the spectrum of $\mathrm{CI} \mathrm{Cyg}$ at minimum was characterized by strong $\mathrm{He} \mathrm{I}$, $\mathrm{He}$ II and Balmer emissions together with rather weak lines of [O III] 4363, [Ne III] 3868 and [Fe VII] 6086. The TiO bands were strong. In 1970, the star was still at minimum, $m_{v} \sim 11.6$ (Lowder, 1971). An infrared spectrum taken with camera VII showed that only $\mathrm{H} \alpha$ and possibly a faint $\mathrm{He}$ I line at $10830 \AA$ were in emission, over a strong continuum of middle $M$ spectral type.

In June 1971 the star brightened to $v=9^{m} \cdot 6$. The spectrum changed slightly, being now characterized by the same lines as in 1969-70 except [Fe VII] and by the increasing strength of the [O III] and [Ne III] forbidden lines. TiO bands were still recorded, but weaker than in 1970 (Figure 2). Grating spectra at $120 \AA \mathrm{mm}^{-1}$ showed in addition the emission line of $\mathrm{O}$ I $8446 \AA$, stronger than the nearby Paschen lines.

From October to December 1971, when the star was at maximum with $m_{v}=9^{m} \cdot 2$ (Isles, 1973), the spectrum changed radically: the nebular lines as well as the permitted lines of high I.P. disappeared, while the lines of Fe II became fairly strong. The Balmer lines were the strongest emission feature in the spectrum. P Cyg effect was observed at $\mathrm{H} \delta$ and possibly at $\mathrm{H} \varepsilon$. While the blue-red bands of $\mathrm{TiO}$ were no 
CI CYG

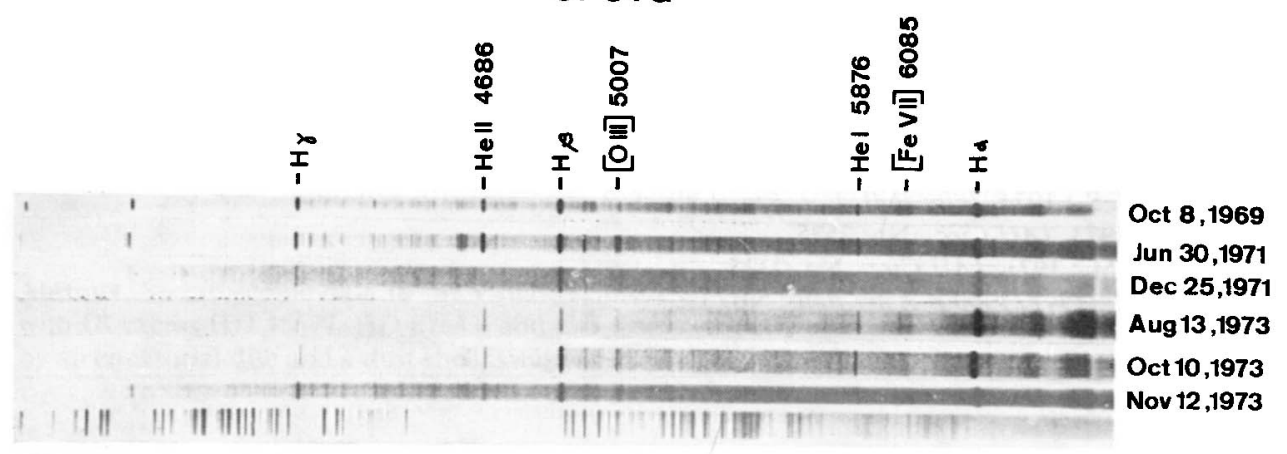

Fig. 2. Spectroscopic evolution of CI Cyg from 1969 to 1973.

longer apparent, the strongest band at $7054 \AA$ was still visible although filled-in by the enhanced continuum.

From May 1972 to August 1973 while the star was declining, the spectrum changed in the reverse way: the TiO bands were becoming strong again; the emission lines of high I.P. were strengthening as well as the nebular lines. Finally even the forbidden lines of [Fe VII] became once more visible. In August 1973 the spectrum became similar to that observed in 1969.

The star underwent a second less pronounced outburst in October 1973 (Belyakina, 1974) again characterized by a lower excitation: He II $4686 \AA$ was weaker than He I $5876 \AA ;$ [ $\mathrm{Fe}$ VII] disappeared. However, P Cyg structures were not seen at this phase and the [O III] lines at 4363 and $5007 \AA$ maintained a moderate strength, as in June 1971. Although the continuum was strong the TiO bands remained conspicuous.

\section{Concluding Remarks}

Detailed data will be published for these two stars in the near future. It may be interesting, however, to note some points at this stage.

The weakness of the nebular lines, together with the absence of the doublets of [O II] 7318-30 $\AA$, [S III] 9069-9532 $\AA$, [A III] 7135-7751 $\AA$ and the [N II] forbidden line at $\lambda 5755 \AA$ may suggest that the nebulae surrounding CI Cyg and $Z$ And are relatively small compared with planetary nebulae where these lines are present together with the lines of [Fe VI] and [Fe VII]. At the same time, the strength of the $\mathrm{Fe}$ II lines imply that they are not emitted in the nebula, but possibly in the atmosphere of the late type star where the TiO bands are also formed. Finally, since the TiO bands are seen even when P Cyg effect is recorded, it can be assumed that the expanding shell surrounds the hot star responsible of the high excitation. This may explain why at the time in which P Cyg structures are seen, neither nebular lines nor $\mathrm{He} \mathrm{I}-\mathrm{He}$ II lines are observed in CI Cyg. Transient shells around the hot component of a binary system imbedded in a small nebula can explain the features observed either in CI Cyg or in Z And. 
In this connection, it may be recalled that Pucinskas (1972) proposed a binary model to take into account the shapes of the minima in the periodic light curve of CI Cygni.

\section{References}

Belyakina, T. S.: 1974, Info. Bull. Var. Stars, No. 863.

Isles, J. E.: 1973, IAU Circ., No. 2575.

Lowder, W. M.: 1971, IAU Circ., No. 2335.

Pucinskas, A.: 1972, Vilnius Astr. Bull., No. 33. 